\title{
The correlation effect between two defects in one-dimensional photonic crystal Lun $\operatorname{Tan}^{1}$, Zhiguo Wang ${ }^{1}$ \\ ${ }^{1}$ Physics department of science and technology,Tongji University, Shanghai, China
}

\begin{abstract}
Keywords:one-dimensional photonic crystal, two defect modes, correlation effect, tunable
\end{abstract} structure

\begin{abstract}
Double defect modes are introduced in one-dimensional photonic crystal. Through studying the correlation effect between the two defects, explored new regularities of the change of the two defect modes with the transfer matrix method. Proposed a new implementation method in researching the photonic crystal with periodic cycles tend to infinity. At the same time, discussed the situations in which the refractive index of the dielectric materials is greater than the existing laboratory conditions, provided theoretical basis for preparation of photonic crystals with new materials in the future.
\end{abstract}

\section{Introduction}

Photonic crystals are composed of periodic dielectric microstructures that affect the propagation rate of photons. The periodic structure can form ban-gaps that restricts the transparency of photons with certain frequencies. This property is extremely useful in both macroscopic ways to fabricate self-cooling covers for energy efficient buildings and microscopic ways to make highly accurate optic experimental equipment. However, if we introduce a defect layer into the periodic layers, a narrow transparent peak will be generated in the crystal's ban-gap. This transparent peak is defined as the defect mode. This article will discuss the correlation effect between two defect modes.

There has been a lot of research on multiple defect modes introduced in one-dimensional photonic crystal. However, most of them are based on limited number of periodic cycles, which causes the ambiguity of defining which layers are genuine layers and which layers are defect layers. What is worse, their conclusion is based on certain types of structures. However, different structure may lead to complete different defect modes. In this article, we introduced a photonic crystal structure type that can be tuned to infinite cycles, which helps the result to converge to be more accurate. In addition, as a result of the increasing popularity of metamaterials, we also discussed materials with refractive index that is higher than any of the materials that can be found in the nature word. This provides a theoretical guidance for future photonic crystal manufacture.

\section{Structure Model and Analyzing}

To approach the infinite periodic cycles, we need to introduce a limited structure first: $(A B)^{M} C(\mathrm{ABA})_{N} D(B A)^{M}$. In this model, $\mathrm{A}$ is $\mathrm{SiO}_{2}$ with refractive indexof $n_{a}=1.45$, and $\mathrm{B}$ is $\mathrm{TiO}_{2}$ with refractive index of $n_{b}=2.3$. The thickness of $\mathrm{A}$ and $\mathrm{B}$ fulfills $n_{a} d_{a}=n_{b} d_{b}=\frac{\lambda_{0}}{4}$, among which $\lambda_{0}=1500 \mathrm{nmis}$ the central wave length. Layer A and B are periodic layers, while C and D are the introduced defect. $\mathrm{M}$ represents the number of cycles, and $\mathrm{N}$ represents the number of layers between the two defect layers. For example, when $N=1$, the layer structure between $C$ and $D$ is (A), when $\mathrm{N}=3$, the structure is (ABA). By increasing $\mathrm{N}$, we can change the distance between two defect layers. To fascinate the representing of results, we only included the situations when $\mathrm{N}$ is odd so that the structure can be symmetric. For asymmetric structures, the result is different, but will not be discussed in this article. By escalating the number of $\mathrm{M}$, we can predict the result of infinite periodic crystals. 


\section{The influence of periotic number $M$ to the defect modes}

To research the influence of $\mathrm{M}$, we set the other parameters of this $(A B)^{M} C(\mathrm{ABA})_{N} D(B A)^{M}$ structure as constants: the thickness and refractive index of $\mathrm{C}$ and $\mathrm{D}$ fulfills $n_{c}=n_{d}=2$ and $n_{c} d_{c}=n_{d} d_{d}=\frac{\lambda_{0}}{4}$. Then, increase M from 2 to 6 . The defect modes are shown as follows:

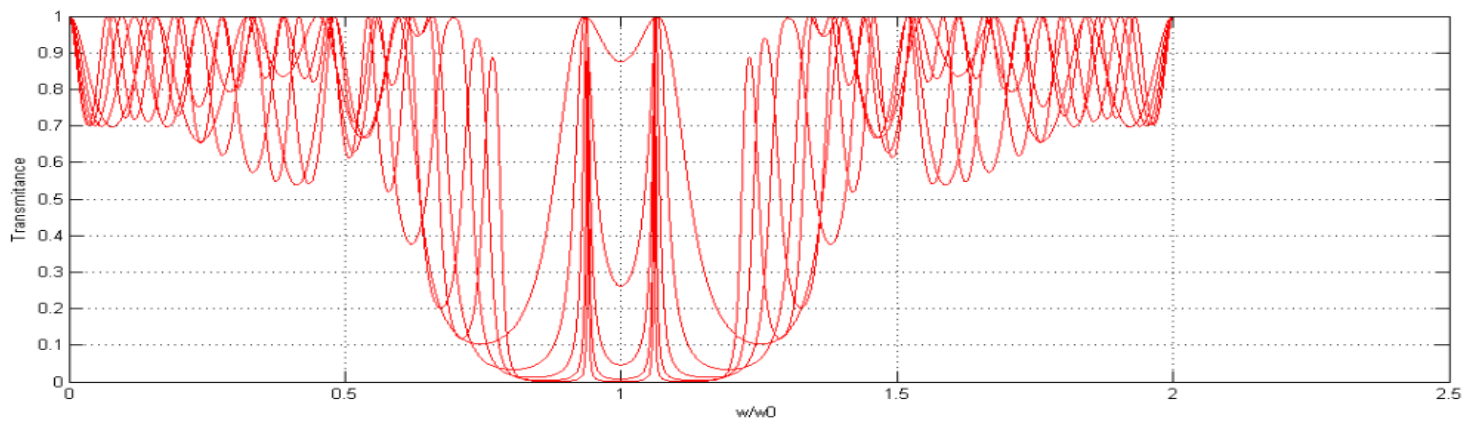

Fig 1. Two defect modes changing with the periotic number $M$

From fig 1, we can know that M does not change the position and the value of defect modes. The value of the two defect modes can always reach 1, and the position of them converges to a final positon. $\mathrm{M}$ only makes the defect modes sheerer, which means the two defect layer can filter photons with specific frequencies more accurately. With this knowledge in mind, we can set $\mathrm{M}=4$ in the next sections and increase it after finding the general rules at $\mathrm{M}=4$.

\section{The influence of distance $\mathbf{N}$ to the defect modes}

Let the periotic number $M=4$, and defect layer $C$ and $D$ have the same parameters as section 1.1. Increase the distance $N$ between $C$ and $D$ as $1,3,5,7,9$, etc. The defect modes are shown as follows:

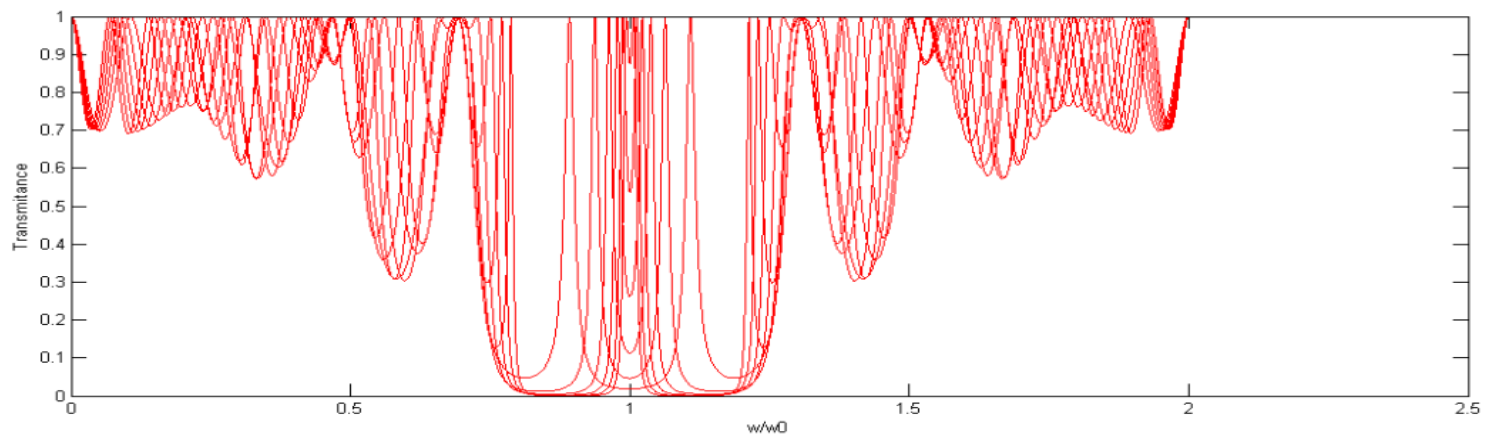

Fig 2. Two defect modes changing with the distance $N$

In fig 2, we can find that the value of two defect modes remains the same, both of them can reach the value of 1 . However, the position of them is getting closer when $\mathrm{N}$ is increased. From this phenomenon, we can conclude that increasing the distance of $\mathrm{N}$ between two defect layers is reducing the correlation effect of the two defect modes because the gap between the two modes positively represents the correlation two layers.

With further simulation, when $\mathrm{M}=4$, and $\mathrm{N}$ is larger than 13 , the two separated defect modes will merge into one smaller defect mode. However, the merged defect mode can become separated again if we add the periodic number $\mathrm{M}$. The reason for this separation is discussed in section 1.1 that the increasing of $\mathrm{M}$ will narrow the width of each defect mode, thus making the result more accurate. Therefore, increasing $\mathrm{N}$ can reduce the correlation effect between two defect layers when the periodic number of the photonic crystal is trending to infinite.

\section{The relationship between two defect modes when the two defect layers are identical}

The rule of the ways that two defect modes changing with the two defect layers is different in two situations: one is when the two identical layers fulfills the requirement of quarter-wave plate, 
the other is when the two layers are not quarter-wave plate. We will research the situation that both defect layers are quarter-wave plates first and then discuss the situation when the two defect layers are identical but not quarter-wave plates.

1. The situation when the two defect layers are identical and quarter-wave plates.

In our photonic crystal structure $(A B)^{4} C(\mathrm{ABA})_{3} D(B A)^{4}$, let the periodic number $\mathrm{M}=4$. The thickness and refractive index of $\mathrm{C}$ and $\mathrm{D}$ fulfills $n_{c}=n_{d}$ and $n_{c} d_{c}=n_{d} d_{d}=\frac{\lambda_{0}}{4}$. Increase $n_{c}=n_{d}$ by 0.25 from 1.0 to 2.25 , the two defect modes are shown in the following figure.

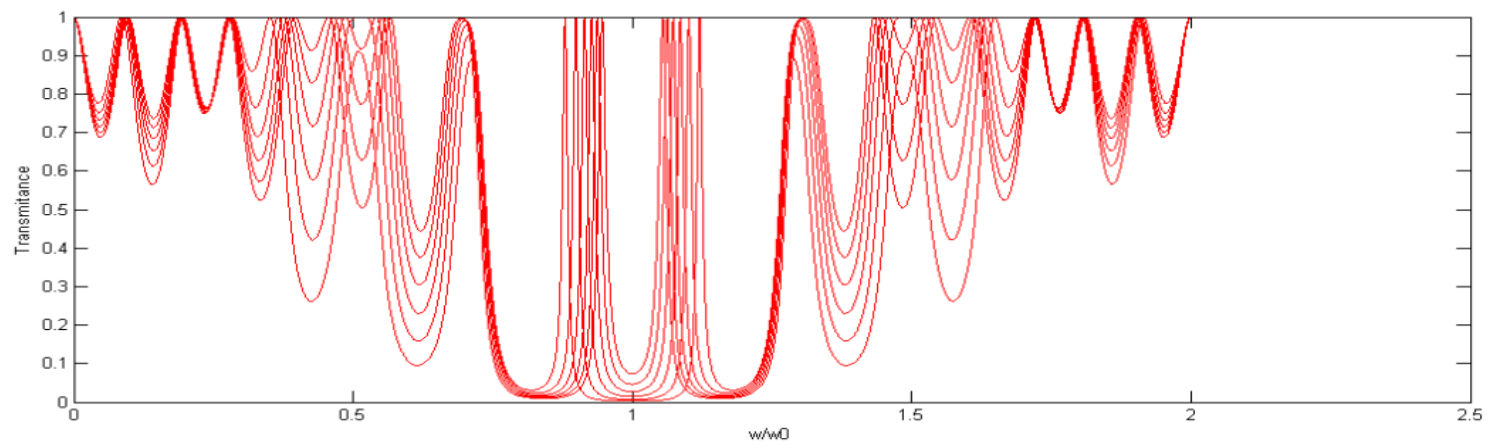

Figure 3(a)

From figure 3(a), we can find that the value of each defect modes also keeps at 1 . At the same time, the two defect modes moved closer when the refractive index was increased, which indicates that the correlation effect between two defect layers isreduced. In the following figure 3(b), if we continue increasing the refractive index, the two defect modes will get closer until they merge into one.

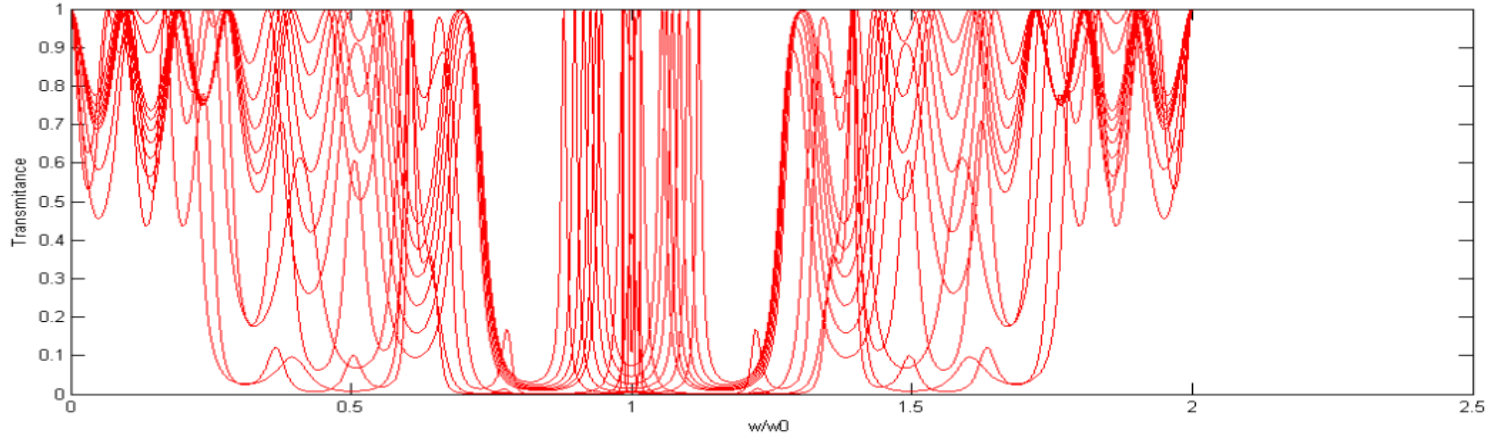

Figure 3(b)

With further inspection, the merged defect mode can also be separated if the period number $\mathrm{M}$ is increased. However, the distance of the two defect modesis still very close, which indicates that the correlation effect is still fragile.

2. The situation when the two defect layers are identical but not quarter-wave plates.

In our structure $(A B)^{4} C(\mathrm{ABA})_{3} D(B A)^{4}$, let the periodic number $\mathrm{M}=4$. The thickness and refractive index of $\mathrm{C}$ and $\mathrm{D}$ fulfills $n_{c}=n_{d}$ and $n_{c} d_{c}=n_{d} d_{d}=\frac{\lambda_{0}}{2}$. Increase $n_{c}=n_{d}$ by 0.25 from 1.0 to 2.25, the two defect modes are shown in the following figure 4.

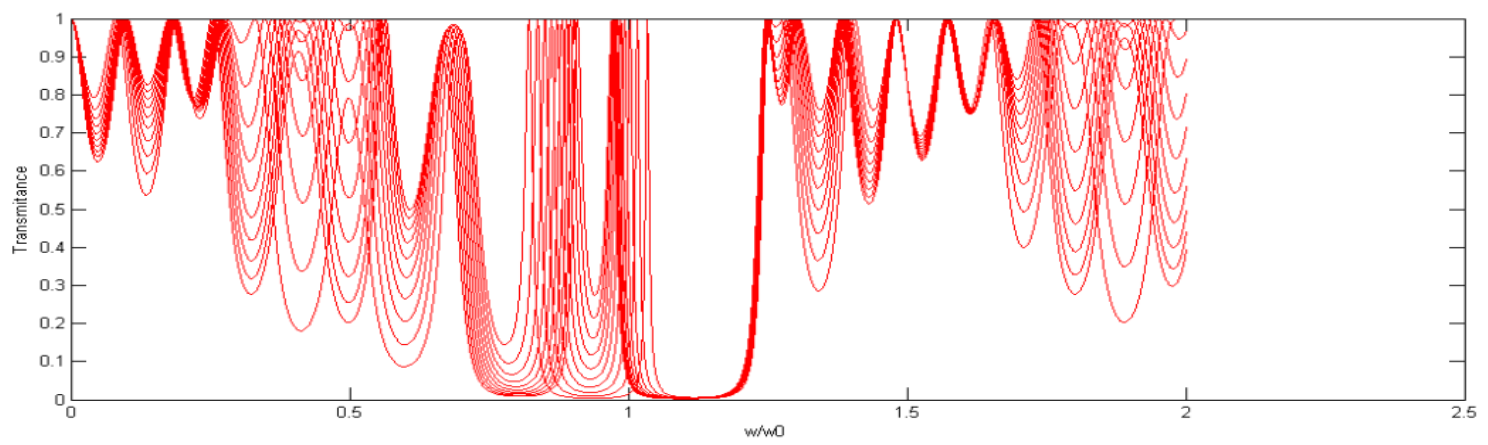

Fig 4. Two defect modes when two defect layers are identical but not quarter-wave plates 
From fig 4, we can know that the position of the two defect modes is no longer symmetric. However, the value of each defect mode is still 1 since the two defect layers are identical.

\section{The relationship between two defect modes when the two defect layers are different.}

From section 4, we know that the two defect modes will be symmetric to the central frequency if both defect layers are quarter-wave plates. In this section, we will continue the inspection on whether this rule is still valid when the two defect layers are not identical. In addition, we will also discuss the situations when the defect layers are not quarter-wave plates.

\section{The situation when two defect layers are quarter-wave plates.}

In our structure $(A B)^{4} C(\mathrm{ABA})_{3} D(B A)^{4}$, keep the parameters of one defect layer $\mathrm{C}$ as $n_{c}=2$, andlet the width of $\mathrm{C}$ fulfill $n_{c} d_{c}=\frac{\lambda_{0}}{4}$ at the same time. The other defect layer $\mathrm{D}$ also fulfills $n_{d} d_{d}=\frac{\lambda_{0}}{4}$. The refractive index $n_{d}$ is increased from 1 by 0.25 to 4.5 . The changing of defect modes is shown as follows:

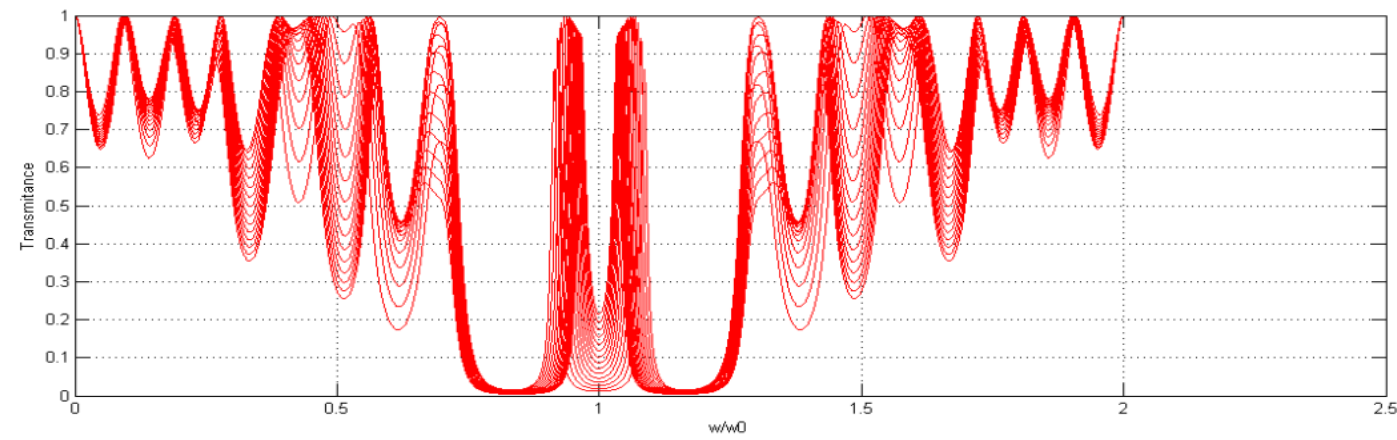

Fig5. Two defect modes when defect layers are quarter-wave plates.

From fig 5, we can know that while two defect modes still have the same value, they are no long able to reach the value of 1 . With the incremental of $n_{d}$, two defect modes move closer to the central frequency. The value of them first increases then decreases. Specifically, when $n_{d}=2$, the two defect modes reached the peak value of 1 because at this time, the two defect layers were identical. This matches the rule discussed in section 4 .

With further inspection that we continue the increment of $n_{d}$, the two separated defect modes will merge into one when $n_{d}$ is larger than 17.As is shown in fig 6 , when $n_{d}=17$ is significantly larger than the periodic layers' refractive index, which is less than 3 , the transparent rate of the whole structure is expressively influenced by this defect layer. However, if we increase the periodical number $\mathrm{M}$ instead, the merged defect mode will still be separated again.

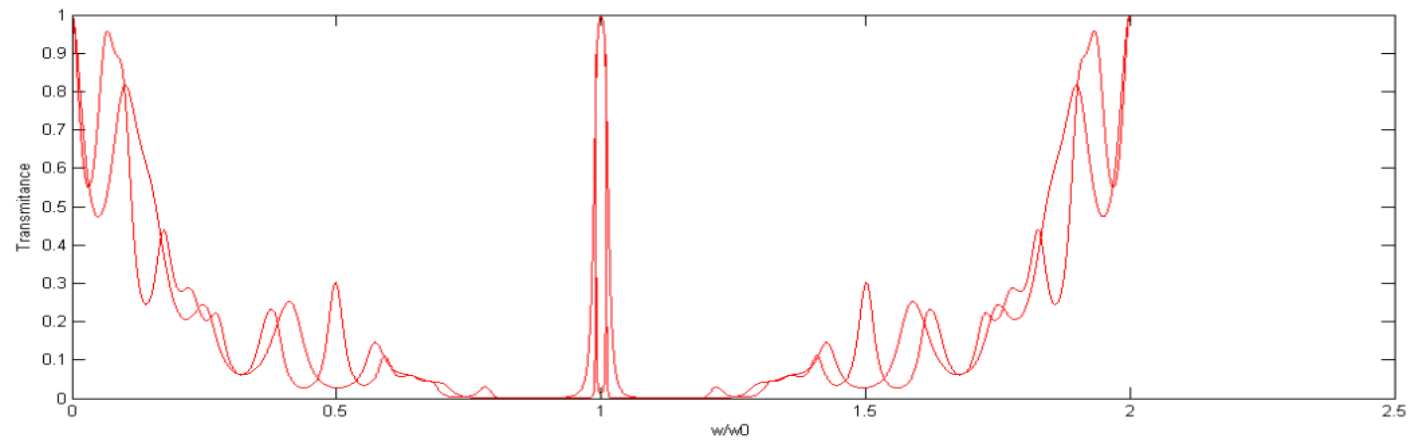

Fig 6. Two separated defect modes merge into one.

\section{The situation when two defect layers are not quarter-wave plates.}

In our structure $(A B)^{4} C(\mathrm{ABA})_{3} D(B A)^{4}$, keep the parameters of one defect layer $\mathrm{C}$ as $n_{c}=2$, and let the width of $\mathrm{C}$ fulfill $n_{c} d_{c}=\frac{\lambda_{0}}{4}$ at the same time. The other defect layer $\mathrm{D}$ fulfills $d_{d}=d_{c}$. The refractive index $n_{d}$ is increased from 1 by 0.25 to 4.5 . The changing of defect modes is shown as follows: 


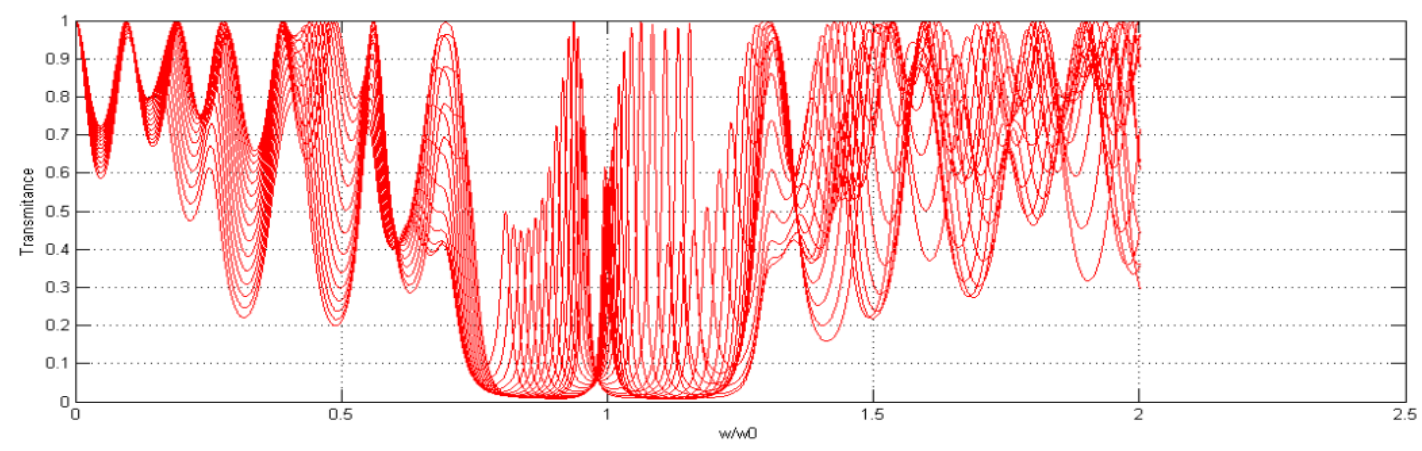

Fig 7. Two defect modes when defect layers are not quarter-wave plates

From fig 7, we can find that the two defect modes are no longer symmetric to the central frequency. In addition, the value of each defect mode is also not identical. With the incremental of layer D's refractive index $n_{d}$, both defect modes move toward the lower frequent state, which indicates that the resonated defect modes tend to have longer optical path.

\section{Conclusion}

According to the theoretical simulations above, for photonic crystals that has the structure with two defect layers $(A B)^{M} C(\mathrm{ABA})_{N} D(B A)^{M}$, five conclusions can be wrapped up. (1), The position of the two defect modes is not affected by the periodic number $M$. Increasing $M$ will only narrow down the width of each defect modes.(2), The correlation effect between two defect layers is more significant when the distance of them is closer.(3), The value of each defect modes is able reach 1 when the two defect layers are identical. On the contrary, if the two defect modes break the symmetrical structure of the whole crystal, the two defect modes cannot reach 1 . (4), The two defect modes are symmetrical to the central frequency if both of them are quarter-wave plates. (5), Keeping one defect layer stable and increasing the refractive index of the other will cause both defect modes moving towards the lower frequency stage. However, the moving speed of the two defect modes is not identical. In conclusion, with the help of tunable photonic crystal structure, the rule of correlation effect between two defect modes is presented with higher precision. In addition, with the potential of metamaterials, the refractive index is no longer a limitation in both practical and theoretical aspects. Therefore, simulating defect layers with higher refractive index than the natural materials is also performed to guide the experimental practice in the future.

\section{Reference}

[1] Guo Shuohong. Electrodynamics [M]. Beijing: Higher Education Press, 2011: 135-140. (in Chinese).

[2] Aaswath P. Raman1, Marc Abou Anoma2, Linxiao Zhu3, Eden Rephaeli1 \&Shanhui Fan1. Passive radiative cooling below ambient temperature under direct sunlight[J]. Nature. 2014(11):540-550.

[3]Bazilian, M. et al. Re-considering the economics of photovoltaic power. Renew Energy 53, 329328(2013).

[4] Li Zhiyuan, Qin Zidong, Tan Lun, Two Designs of Thin Film for cooling Buildings based on Photonic Crystal. AER-Advances in Engineering Research 2015(2): 95-98.

[5] E. Yablonovitch, Inhibited spontaneous emission in solid-state physics andelectronics, Phys. Rev. Lett. 58 (1987) 2059-2062.

[6] H.F. Zhang, S.B. Liu, X.K. Kong, L. Zou, C.Z. Li, W.S. Qing, Enhancement of omnidirectional photonic band gaps in one-dimensional dielectricplasma photonic crystals with a matching layer, Phys. Plasmas 19 (2012)022103-1-022103-7.

[7] Srivastava SK, Ojha SP. Enhancement of omnidirectional reflection bands inone-dimensional photonic crystals with left-handed materials. Prog Electro-magn Res 2007;68:91-111.

[8] E. Cojocaru, Electromagnetic tunneling in lossless trilayer stacks containing single-negative metamaterials, Prog. Electromagnetics Res. 113 (2011)227-249. 